\title{
Cantos funerarios en Colombia y Venezuela. Su rescate a través de la formación docente
}

\author{
Funeral songs in Colombia and Venezuela. Your rescue through Teacher Training
}

\author{
Christian Andrés Hernández Rodríguez \\ christianandreshernandez@gmail.com \\ Código ORCID: 0000-0001-8364-5178 \\ Universidad de San Buenaventura Cali, \\ Colombia
}

- Marlene Arteaga Quintero

marlenearteagaquintero@gmail.com

Código ORCID: 0000-0003-3370-2829

Universidad Pedagógica Experimental

Libertador, Miranda, Venezuela

- Alexánder Amézquita Pizo

alexpizo@gmail.com

Código ORCID: 0000-0000-0001-5752-6132

Universidad del Valle, Colombia

| Artículo recibido en febrero 2020 | Arbitrado en abril 2020 | Publicado en mayo 2020

\section{RESUMEN}

Palabras clave:

Alabaos; cantos funerarios; cultura afroamericana; transversalidad; literatura
En las Instituciones de Educación Superior de Colombia y Venezuela, países estrechamente relacionados por su cultura e historia, existen programas de Literatura y Lengua Castellana que guardan similitudes. En Venezuela y en la Universidad de San Buenaventura Cali de Colombia, los estudiantes de docencia en sus programas de la especialidad podrían tener la oportunidad de aprehender parte de la cultura afroamericana como un eje transversal más que como un contenido conceptual. Con esta orientación, se revisó la manifestación de Los alabaos en Colombia, se consultó a tres expertos de la costa del Pacífico colombiano y a tres expertos venezolanos. Con la revisión de los contenidos y testimonios se propone el rescate de los cantos funerarios dentro de las propuestas pedagógicas del Plan de Estudios de la USB de Cali, especialmente. Con ello, los educadores podrán abordar saberes esenciales dentro del entorno académico e integrarlos a sus aulas de clase.

\section{Keywords:}

Alabaos; funeral songs; African American culture; transversality; literature

\section{ABSTRACT}

In the Institutions of Higher Education of Colombia and Venezuela, countries closely related by their culture and history, there are Literature and Spanish Language programs that have similarities. In Venezuela and at the University of San Buenaventura in Cali, Colombia, teaching students in their specialty programs could have the opportunity to learn part of African-American culture as a transversal axis rather than as a conceptual content. With this orientation, the manifestation of the Alabaos in Colombia was reviewed, three experts from the Colombian Pacific coast and three Venezuelan experts were consulted. With the revision of the contents and testimonies, the rescue of the funeral songs is proposed within the pedagogical proposals of the Curriculum of the USB of Cali, especially. With this, educators will be able to approach essential knowledge within the academic environment, and integrate it into their classrooms. 


\section{INTRODUCCIÓN}

Existen una serie de manifestaciones culturales propias de la costa pacífica de Colombia que son parte de las producciones orales de los grupos más arraigados en el territorio. Estas responden, siguiendo a Moreno Blanco (2008, pp. 90 - 96), a los caracteres fundamentales de este tipo de creaciones: no son textos de autor, podrían eventualmente convertirse en escritos, son de género variado, pertenecen a una tradición inmemorial, y las modificaciones son frecuentes debido a su propio carácter de propiedad comunitaria.

En la producción oral del Pacífico colombiano es fundamental tener en cuenta la influencia de tres culturas (Amézquita y Hernández, 2013): la tradición europea, el legado de los esclavos africanos y los saberes indígenas. Esta dote constituye el mundo oral del Pacífico colombiano y de otros territorios de todas las Américas: allí en donde se vacían un universo de experiencias vitales, memorias y evolución de los imaginarios culturales. En este contexto han surgido manifestaciones como las décimas, los romances, las oraciones, las retahílas o ensaladillas, las coplas, las leyendas, los mitos y fantasmagorías, las narraciones históricas, las narraciones didácticas, los juegos, las loas, las adivinanzas, los arrullaos o cantos de cuna, los salves o alabanzas de pasión, los chagualos, los gualíes o cantos de angelito, las rondas, los cantos de boga y los alabaos (Puertas Arias, 2000).

En su descripción, Motta González (1997) ubica los alabaos como una manifestación cantada por voces femeninas, preferentemente; Prado (1996), los sitúa como producción rimada y cantada. Su expresión con rima, bajo códigos orales, con patrones hispánicos, ritmo africano e indígena y su pervivencia en la memoria como patrimonio inmaterial relevante.

La intención, en esta propuesta, no es estereotipar el mundo de lo afro ensimismado en la danza, el tambor, el canto y la recitación; no se pretende determinar lo afroamericano como solo música y religiosidad dejando a un lado sus aportes a la ciencia, las artes, la filosofía o la educación (Fernando Montaño, bailarín; Libia Grueso, ambientalista; Raúl Cuero, biotecnólogo; Doris Hinestroza, matemático). El propósito es rescatar la condición esencial de los alabaos como un monumento cultural afrocolombiano dentro de los estudios literarios, en conjunto con la formación docente.

Para los profesores universitarios, principalmente aquellos quienes forman a los especialistas en Literatura y Lengua Castellana, estas manifestaciones tienen especial valor debido a su riqueza lingüística y literaria y a su trascendencia históricocultural. Por consiguiente, debe ser una tarea inaplazable incluir parte de esta herencia propia de las comunidades afro dentro de los programas curriculares para la formación docente.

En consecuencia, luego de estudiar las teorías, participar de experiencias en el campo, revisar los programas de estudio, surgen los interrogantes: ¿podrían los expertos ofrecer sus conocimientos de los alabaos para incrementar las perspectivas en esta investigación? ¿Podrían compararse, sucintamente, las visiones de conocedores de Colombia y Venezuela en función de las semejanzas y las necesidades de formación? ¿Tienen los cantos funerarios como los alabaos un valor cultural que puede ser rescatado por la escuela? ¿Podrían ubicarse de forma transversal en los planes de estudio para los docentes? 
El objetivo, entonces, de esta indagación, es proponer la inclusión del eje transversal Representaciones afroamericanas dentro del Plan de Estudios de la Universidad de San Buenaventura Cali, a partir de una investigación, en particular, sobre los alabaos (como ejemplo de las múltiples manifestaciones existentes). La aproximación se realiza desde la revisión del tema de los cantos funerarios, la entrevista abierta con expertos colombianos y la técnica de la entrevista dirigida a expertos venezolanos. $\mathrm{La}$ experiencia llevó a considerar que toda la herencia hispánica, indígena y africana debe formar parte del acervo cultural y educativo de toda la comunidad, mediada a través de la intervención de sus docentes, por lo que en esta oportunidad se trabaja sobre el rescate de las "huellas de africanía" presente en los alabaos, como diría Friedemann (1992, 1997).

Los alabaos se definen como cantos a los muertos que se realizan en "los velorios, en las novenas de los santos y en el último día del novenario para despedir a un difunto. Alabao viene de alabanza, concretamente, alabanza a Dios" (Amézquita y Hernández, 2012). La composición se transforma en un diálogo y un canto dedicado a honrar a los santos, a la Virgen, a Dios, al difunto y a su familia y su comunidad. Estas comunidades pertenecen a todo el Pacífico colombiano, desde el Chocó hasta Nariño, especialmente en los estratos más pobres, los asentamientos populares y las zonas rurales en donde se conserva la tradición. En los barrios de Quibdó, en las urbanizaciones de mejor condición socioeconómica, en las comunidades pudientes estas prácticas ya casi nunca forman parte de su cultura y se han perdido casi por completo con las influencias externas.
Los ritos fúnebres de los alabaos se realizan durante nueve días, en los que se distinguen seis momentos (Tabla 1): agonía, muerte, velorio, entierro, novena y última noche. Las mujeres, sobre todo, cantan y lloran, pero no se excluye a los niños y niñas que aprenden con el ejemplo que presencian como parte de su herencia.

Cuando una persona del Pacífico negro se encuentra grave, la comunidad se prepara por solidaridad a realizar todo el ritual mediante un construir colectivo en donde confluyen el dolor, lo popular, la fiesta, lo religioso en una narración de lo sagrado:

El velorio no es entonces una
muestra de dolor solemne, sino una
celebración con cantos, juegos,
episodios narrativos, libaciones y
cierta alegría latente, para exaltar la
grata memoria del personaje
fallecido. El sincretismo religioso
hace esfumar un tanto las nociones
convencionales de cielo e infierno, o
del demonio que castiga a los
males, y las remplaza por un
concepto de supervivencia mucho
más funcional y apropiado a la
conservación de las tradiciones del
grupo social. (Marulanda, como se
citó en Tobón, 2010, p. 43)

El primer momento se conoce como "la agonía", cuando las mujeres comienzan con los cantos en la víspera de la muerte de un enfermo. Con ese aviso llegan los vecinos y comienzan los primeros alabaos en donde se incorporan los rezos del Padre Nuestro, el Credo, la Salve. La Corporación Centro de Pastoral Afrocolombiana de Buenaventura, refiere que los asistentes rodean al moribundo y le ofrecen oraciones en susurros, además de persignarlo. El segundo momento se conoce como "la muerte". Al 
finado las mujeres lo visten y preparan en medio de gritos y rezos pidiendo a Dios por su alma. Suele cantarse el alabao en la versión de Madolina Palacios, registrado por Córdoba y Rovira (2003, p. 63). El tercer momento se conoce como "el velorio" en donde se come, se bebe y se juega en comunidad. Hombres y mujeres (Córdoba y Rovira, 2003) rezan tres rosarios: uno a las 6:00 pm, otro a las 12 de la noche y otro a las 6:00 am del día siguiente (Tabla 1).

El cuarto momento "el entierro" se ocurre en el camino al cementerio con una peregrinación entusiasta, bulliciosa y desgarrada acompañada del rosario y del canto de alabaos. El quinto momento es "la novena" nueve días de rezos "para pedir la purificación y limpieza del alma del difunto y el perdón de sus pecados" (CEPAC, 2010, p. 32), las velas encendidas por solo 1 hora. Se cantan alabaos que irán aumentando de a uno, día tras día hasta el noveno día. El sexto momento se denomina "la última noche". Todos los asistentes cantan y rezan fervientemente tres rosarios: uno a las 8:00 pm, otro a las 12 de la noche y otro a las 5:00 am antes de que amanezca y que llegue la luz del día; se desmonta lo que denominan "la tumba" y el resto de las velas con un avemaría y un último alabao (Tabla 1).

Debe anotarse que en esta región el proceso de colonización estuvo marcado por el lavado de oro y al no ser suficientes los indígenas se requirió la mano de obra negra. En los campamentos mineros iniciales los nativos y los hombres esclavizados recién llegados compartían sufrimientos, y los primeros empezaron a transmitir sus conocimientos en la exploración fluvial y en la fabricación de canoas. Esta relación interétnica se va a fracturar una vez que se empiece a legislar sobre la protección de los grupos nativos, pero el intercambio de símbolos se había operado en gran parte. (Losonczy, 2006).

Con el paso del tiempo y después del asentamiento definitivo de los afrodescendientes en esta región, la relación entre etnias se afianza especialmente en el ámbito comercial, sin embargo, es indudable que cada cultura ha recibido la influencia de la otra. Mientras que los indígenas proveen a los negros de elementos hortícolas, productos artesanales como canoas, cestas, entre otros; los afrodescendientes funcionan como intermediarios de productos que se encuentran en el exterior: sal, fósforos, machetes, hachas, escopetas, combustible y aguardiente de fabricación local. Lo más interesante de este intercambio es que se realiza principalmente durante épocas en las que se celebran festividades en las poblaciones afrocolombianas: la Semana Santa, la de San Antonio y la Navidad, además del carácter comercial por tanto los indígenas son invitados a participar de dichas celebraciones.

Otro momento del año en donde las distintas etnias conviven se da durante el período de siembra y cosecha de maíz, los negros acuden a los indígenas para que les apoyen, pues su principal ocupación es la explotación y lavado de oro. Al respecto Losoncky (2006) ha establecido una serie de vínculos, tanto de los afrocolombianos como de los Emberá, correspondiente a los rituales de paso, entre ellos, entre el que corresponde al de los ritos fúnebres, en los que, como sabemos juega un papel fundamental la presencia de los alabaos.

La antropóloga caracteriza los Alabaos como:

los cantos funerarios constituyen la palabra misma del alma del muerto, dicha por la voz de los vivos. Cantar un alabao significa entonces que 
este espíritu, en el eje de los dos mundos, debe ser acogido en el interior del cantante, allí donde el alma - sombra propia deja la huella de la memoria y el lenguaje. (Losonczy, 2006, p. 223)

Como se observa, aun bajo la traza antropológica, la expresión lírica es el fundamento discursivo de estas manifestaciones, sobre todo organizadas en estructuras estróficas procedentes de Europa. Estas expresiones literarias se hicieron presentes de diversas formas, pero fueron la Décima y el Romance, las que mayor arraigo tomaron en el Pacífico (Prado, 1996).

La décima es la expresión popular que más fuerza tiene en el Pacífico colombiano, y según la investigadora Puertas Arias (2000), esta región adoptó la forma glosada. Esta manifestación, a pesar del tiempo ha conseguido conservar su modo de rimar, los cambios que se han presentado, especialmente, se dan en el plano del léxico local. Sin embargo, si se desea encontrar una influencia contundente en estas expresiones rituales, es evidente que el proceso de evangelización al que fueron sometidos los pueblos americanos y los hombres esclavos traídos de otros continentes, es el factor que más consiguió permear las prácticas fúnebres.

Los rituales africanos no desaparecieron en el momento de la imposición católica por parte de los europeos, sus diferentes dinámicas consiguieron sobrevivir el paso del tiempo. Los santos católicos jugaron un papel fundamental en el proceso de transculturación que se dio en el Pacífico colombiano, pues, aunque fueron impuestos, poco a poco se involucraron en las distintas actividades del diario vivir. Los afrocolombianos consiguieron reconfigurar las funciones de los santos católicos, por eso se les trata de dos formas: la primera marcada por el santoral católico, que exige un comportamiento de subordinación frente al santo y otra más flexible, en donde el santo se convierte en uno más de la comunidad. (Ayala Santos, 2010, p. 37)

Los santos, son irremplazables en especial aquellos

que han sido cargados de sentido a través de las tradiciones orales y los usos prácticos (San Antonio, La Inmaculada en Guapi, el señor de la Buena Esperanza en Callelarga, la Virgen del Carmen, San Buenaventura). Los santos concitan a su alrededor las fuerzas del inframundo y supramundo, los imaginarios tradicionales oralmente gestionados, la religiosidad católica, la idolatría y mística en torno al monumento, y por supuesto el dramatismo y la fiesta religiosa, el encantamiento / intensificación permanente del mundo de la vida. (González, 1997, p. 21)

Por otra parte, deben considerarse el conocimiento de romanceros, coplas y décimas que provienen de la tradición española. Los alabaos privilegian los versos octosílabos, la décima y los romances. Especialmente, se utiliza la espinela de rito o improvisada y las rimas son múltiples y combinadas (Córdoba, 2014). Todos estos son expresiones literarias que suelen ser abordadas en diversos niveles de la educación sea primaria, secundaria 0 superior, especialmente cuando se tratan temáticas referentes al género lírico.

En los planes de estudio de Literatura y Lengua Castellana se incluye el conocimiento de la historia de la literatura, los contenidos de la literatura española, literatura colombiana, el análisis literario, entre otros, por lo tanto, sería de gran utilidad incluir de forma transversal las Representaciones 
afroamericanas que contemplarían -en principio- el estudio de las estructuras estróficas, los versos, las rimas y los temas propios de toda manifestación literaria con herencia afro, entre ellas los alabaos con su historia, su práctica y su análisis.

\section{MÉTODO}

El trabajo se inscribe dentro del paradigma interpretativo, con un enfoque cualitativo bajo el diseño una investigación de campo y una modalidad de Proyecto Especial (UPEL, 2016, p. 19). La intención es crear una propuesta que satisfaga una necesidad que no requiere un diagnóstico debido a que la creación que se desprende de las revisiones documentales y prácticas es susceptible de ser empleada para necesidades e intereses de tipo educativo.

Su direccionalidad es pedagógica debido a que la metodología interviene el diseño curricular y aspira por tanto tener una utilidad demostrable y tangible para los integrantes del sistema, aun cuando sea en una escala restringida. Además, como lo afirman Ruay, González y Plaza (2016) "una innovación curricular está constreñida por las diversas culturas institucionales, tanto internas como externas, con las cuales hay que dialogar" (p.157). Y precisamente, se pretende, es un intercambio signado por la transversalidad en el currículo.

La interpretación de la realidad parte de la comprensión de la situación contextualizada en la propia localidad. El conocimiento es inductivo en la medida en que se revisan los documentos, se observan los hechos y se interactúa con los sujetos participantes para llegar a conclusiones válidas y proyectos sólidos. Igualmente, "los investigadores siguen la pista de los temas emergentes, leen sus notas de campo o transcripciones y desarrollan conceptos y proposiciones para empezar a dar sentido a sus datos" (Taylor y Bodgan, 2002, p. 158).
Luego de la revisión documental, se aplicó una entrevista semiestructurada a tres expertos colombianos (Amézquita y Hernández, 2012) y una entrevista guiada o dirigida a tres expertos venezolanos. Para recoger la información se utilizó una entrevista de tipo dirigida (Monje, 2011) que fue aplicada (validada por expertos, previamente) a informantes que cumplieran con el perfil de docentes a la vez que expertos en diversas áreas de la cultura: educación musical, antropología, folclore, danza tradicional, canto, entre otras. Esta entrevista cuenta con una información previa que suministra contenido sobre el tema a tratar y posteriormente se realizan tres interrogantes abiertas.

Los expertos entrevistados por Colombia fueron: (a) María Celedonia Olave Mondragón, Directora de la Colonia del Alto Naya, docente y cantadora; (b) Baudilio Revelo Hurtado, abogado, investigador temas afrodescendientes, autor de Derecho y la cultura afrocolombiana del Pacífico, Cofundador y docente de la Universidad Libre de Cali y (c) Alberto Vallejo, artista, investigador de arte y Curador del Museo de Arte Religioso de Cali. Los especialistas de Venezuela fueron (1) la profesora de danza, experta en danzas tradicionales, bailadora, coreógrafa y docente universitaria Yaritza Tineo; (2) la cantautora, percusionista, cultora, docente de Educación Musical e investigadora de la música tradicional venezolana Eva Nazareth Daza y (3) el cantautor, escritor, poeta, Antropólogo, Especialista en Folklorología y Etnomusicología, Magíster en Educación, mención Currículo y Doctor en Educación, Yván Pineda Monasterios.

\section{RESULTADOS}

De la entrevista semiestructurada, realizada por Amézquita y Hernández a los expertos colombianos se rescatan, para esta propuesta, lo siguiente: 


\section{Entrevista (a) María Celedonia Olave Mondragón respondió:}

Interrogante 1: ¿Qué es un alabao?

Un alabao es una alabanza que se hace a Dios nuestro Señor, para establecer una relación entre él y los hombres en unos momentos específicos. Concretamente los alabaos se llevan a cabo en rituales o ceremonias como velorios, entierros, novenas, entre otros. Yo le puedo decir que los alabaos son una fortaleza a la espiritualidad del hombre y la mujer afro. Ya que nosotros cantamos alabaos para despedir a nuestros muertos y darle gracias al Señor. Además, para que nuestros familiares intercedan por nosotros ante nuestro señor Jesucristo, para cuando nos llegue la hora de partir a la eternidad.

Interrogante 2: ¿En qué regiones del país se cantan alabaos?

En toda la región del Pacífico colombiano se practican los alabaos. Es cultura ancestral. Heredado de nuestros ancestros africanos que llegaron en los barcos negreros a América y se fueron estableciendo en los Palenques cercanos a Cartagena de Indias, donde eran recibidos por San Pedro Claver; y así se fueron dispersando por nuestro territorio. Es decir, en todos los lugares de Colombia, donde hay asentamientos negros, existen prácticas como los alabaos y ritos fúnebres cantao's.

Interrogante 3: ¿Y cómo ve a las nuevas generaciones de afrodescendientes?

Hoy, algunos jóvenes tratan de conservar su tradición. He visto que muchos de ellos, que han nacido en la ciudad pero que sus padres o abuelos pertenecen a algún pueblo del Pacífico; regresan a la tierra de sus parientes y tratan de celebrar las ceremonias tal como lo hacían sus abuelos. Esto me parece que es fundamental pues fortalece la tradición. Sin embargo, existe una gran comunidad de hombres y mujeres afros que viven en las grandes ciudades y que no conocen nada sobre estos temas, ellos aún son una población por reconocerse.

\section{Entrevista (b) Baudilio Revelo Hurtado respondió:}

Interrogante 1: ¿Qué es un alabao?

Alabao viene de alabanza. Alabanzas que se hacen a Dios y su origen es africano. Alabao también quiere decir cantos de lágrima, llantos de lágrimas que vienen desde el Luango, en Angola. Pues en la diáspora africana llegaron a América (...) millones de hombres y mujeres cargados de tradiciones, prácticas y así, con lo poco que tenían en sus memorias, comenzaron a honrar a sus muertos con cantos, lamentos que durante nueve noches se cantan por el sufrimiento que produce la muerte de un ser querido. Con el paso del tiempo y la Colonia, el imperio español y la religión católica hicieron que muchos de esos cantos se convirtieran en rezos cristianos. Sin embargo, es claro que su esencia es eminentemente africana.

Interrogante 2: ¿En qué regiones del país se cantan alabaos?

Todo el Pacífico colombiano está habitado de descendencia africana. Desde Panamá hasta el río Mira en Tumaco. En todo este territorio se cantan alabaos. De pronto en el Chocó la musicalidad del alabao sea más alegre que en el sur, donde el alabao es lúgubre; pero conserva las mismas intenciones y sentimientos. En todos los casos el alabao viene acompañando rituales, representación de la solidaridad y espiritualidad entre los pueblos. 
Interrogante 3: ¿Cuál cree que es la importancia de estas prácticas en la actualidad?

Hoy las tradiciones negras están cada vez más perdidas, porque estas personas son obligadas por la violencia y la pobreza a migrar a ciudades como Cali donde llegan a mendigar y es así como pierden toda su espiritualidad y llegan a sentir vergüenza de lo que son y de sus tradiciones, porque una de las razones es que para poder ser aceptados por esta nueva comunidad deben adaptarse a un pensamiento que es extraño a nosotros. Porque nuestro lenguaje es eminentemente oral. Nos expresamos a través de los gestos, con lo que le damos fuerza a nuestra expresión. Por eso creo que estas prácticas son fundamentales, para que no pierda la tradición.

\section{Entrevista (c) Alberto Vallejo}

Interrogante 1: ¿Qué es un alabao?

Es un canto a la memoria de un ser querido que ha partido. Básicamente es esto. En lo que percibí en Chocó, es que ellos lo cantan del alma, lo sienten en su interior y lo expresan en sus tonos graves, agudos y en el movimiento de su cuerpo. Es difícil uno describir algo simplemente con leerlo. Creo que para saber qué es un alabao hay que sentirlo, hay que estar al lado de estas comunidades. Yo me llevé una gran sorpresa cuando estuve al frente de alguien que canta un alabao.

Interrogante 2: ¿Ha habido cambios y aportes en los alabaos?

Es indudable que se ha dado una evolución en las prácticas de estas comunidades. Se da en las nuevas generaciones que están siendo influenciadas por otros ritmos. Aunque los adultos aún lo conservan ellos tratan de que sus hijos también se apropien de él. Esperamos que esto suceda y que las otras dinámicas, las de la ciudad, no sean más fuertes. Por ejemplo, el tema de la funeraria es preocupante.

Interrogante 3: ¿Cuáles son los cambios recientes en los cantos fúnebres?

Sí hay diferencias. Allá en el Pacífico aún se realiza de forma tradicional. Pero aquí en Cali hay algunas características que se han visto modificadas como la comida, pues allá es austero en cuanto a los elementos. Aquí en Cali se reúnen a puerta cerrada, es como un funeral bailable que se presta para otras circunstancias.

Posteriormente, se realizó la entrevista a los especialistas venezolanos. Se configuró de la siguiente forma: 
Christian Andrés Hernández Rodríguez, Marlene Arteaga Quintero y

Alexánder Amézquita Pizo

Tabla 1. Instrumento. Entrevista dirigida

\section{Entrevista-dirigida para experto}

Esta entrevista se realiza con el propósito de indagar si usted conoce, ha participado o ha presenciado alguna experiencia similar a la que se expone a continuación.

Sus respuestas, a las preguntas anotadas al final, serán de utilidad para comparar el ritual de Los Alabaos propio de la costa del Pacífico colombiano y algunos ritos de Venezuela desde la visión de un docente investigador y artista creador, como es su caso.

¡Gracias por su colaboración!

Nombre: Especialidad

\section{PRIMERA PARTE: INFORMACIÓN SOBRE LOS ALABAOS*}

Los alabaos son cantos a los muertos. Tienen lugar en los velorios para despedir a un difunto. Son composiciones dialogadas y cantadas generalmente rimadas que se emplean para honrar a los distintos santos, a la Virgen María, al finado y a la misma comunidad que lo despide. En todo el Pacífico negro colombiano el alabao se practica en los estratos populares y las zonas rurales.

\section{LOS SEIS MOMENTOS DEL ALABAO}

El Alabao se extiende durante nueve días, que suelen dividirse en seis momentos: agonía, muerte, velorio, entierro, novena y última noche. La vocería la llevan los adultos, especialmente las mujeres, pero los niños y niñas están siempre presentes, escuchando, aprendiendo a través del oído cómo es esa toma de la palabra por parte de los adultos.

\section{Primer Momento: La Agonía}

Las mujeres comienzan con lamentaciones por el enfermo en su fase terminal. En la agonía se da el primer grito, el primer canto de lágrimas, para convocar a vecinos, amigos y familiares y para que el barrio se entere de que se acerca el momento de la muerte. Se inician los primeros alabaos que incorporan alabanzas a las divinidades cristianas cantadas con voces caídas, extendiendo las vocales y guiadas por una voz líder.

Segundo momento: la muerte

Cuando fallece, el cadáver es preparado por las mujeres mientras se ofrecen llantos y lamentos, llaman al muerto por su nombre y recuerdan sus buenas acciones con la solicitud al Altísimo para que lo acoja a su lado.

Tercer momento: el velorio

Los vecinos, amigos y familiares entregan comida, licor, juegos, café, velas y lo necesario para comer y beber juntos. Después, las mujeres preparan al fallecido dentro de casa, mientras los hombres esperan afuera, donde beben aguardiente y juegan dominó y otros juegos de mesa. El licor ayuda a aclarar la garganta de las cantaoras y rezanderas para que la voz viva. El primer rezo es un rosario a las seis de la tarde, luego se repite a la media noche y a las seis de la mañana. Este se acompaña con otras oraciones o letanías que solo conocen las rezanderas.

\section{Cuarto momento: el entierro}

Camino al cementerio, en una procesión calurosa, festiva y al mismo tiempo dolorosa, se va rezando el rosario y entonando alabaos. Se recorren los lugares más frecuentados por el difunto: casas de amigos, familiares, el lugar de trabajo, entre otros. En el cementerio, el ataúd es rociado con agua bendita lanzada en forma de cruz y los acompañantes bajan el muerto a la tierra. Acto seguido, las rezanderas entonan el Credo y los familiares echan la tierra sobre el cajón mortuorio llorando y evocando a su difunto. 
Quinto momento: la novena

\section{Entrevista-dirigida para experto}

Durante nueve días se reza por una hora, tiempo que duran las velas encendidas. Después, se narran cuentos y se cantan alabaos, que irán aumentando de a uno, día tras día hasta el noveno día. No se aceptan cuentos bailados. Se dice que "un buen cuento hasta al muerto hace reír y un buen alabao hasta al que no es doliente hace llorar" (CEPAC, 2010, p. 32-33).

\section{Sexto momento: la última noche.}

La organización del último día es un trabajo conjunto entre familiares, vecinos y amigos. La asistencia es mayoritaria. Se rezan tres rosarios, uno a las ocho de la noche, otro a la media noche y el último a las cinco de la mañana. Finalizado el tercer rosario, se apagan las luces y se levanta la tumba conformada por la cruz, los arreglos y lo que quede de las velas, mientras se reza un avemaría, todo ello antes del amanecer. Quien va levantando la tumba le entrega a cada familiar una a una todas sus partes. Este rito propicia que el alma del difunto pueda despedirse de este mundo antes de que llegue la mañana y descanse en paz. También, se acostumbra recitar algunos estribillos de alabaos reconocidos por la comunidad, con un canto colectivo donde incluso llegan a participar los más pequeños, algunos integrantes de las comunidades indígenas cercanos a la familia o forasteros.

* Información tomada y adaptada de Amézquita y Hernández (2012, 2013)

\section{SEGUNDA PARTE: INTERROGANTES}

Interrogante 1: ¿Podría explicar si existe en su comunidad o región algún ritual semejante a los alabaos del Pacífico negro colombiano, así como sus pasos, momentos y asistentes?

Interrogante 2: ¿Podría narrar, brevemente, su experiencia si ha participado en un ritual semejante, además de describir sus pasos, momentos y asistentes?

Interrogante 3: Desde su punto de vista como docente investigador y artista creador ¿podrían incorporarse estas manifestaciones culturales en algunos de los niveles educativos y bajo qué tipo de acciones?

Fuente: elaboración propia

\section{Respuestas a las entrevistas de los especialistas venezolanos}

\section{Entrevista a venezolana (1) Yaritza Tineo}

Interrogante 1: ¿Podría explicar si existe en su comunidad o región algún ritual semejante a los alabaos del Pacífico negro colombiano, así como sus pasos, momentos y asistentes?

Si existen varios rituales semejantes en nuestro país. La hamaca es una manifestación cultural de una tragicomedia que comienza con representación de la muerte de un miembro muy importante y querido del Barrio
San Millán de Puerto Cabello del estado Carabobo de nuestra costa venezolana. Primer momento: La muerte y preparación del difunto: ocurre el lunes de Carnaval antes de la media noche. En la casa del enfermo de muerte, cierran la calle con un precinto de seguridad o listón amarillo que dice "no pase" para que las personas no tengan acceso a la casa donde está muriendo el hombre. Mientras esto ocurre todas las personas esperan detrás del precinto de seguridad entre incertidumbre, algarabía, anécdotas, bebidas y expectativas. El hombre muere, lo preparan en la hamaca y esperan hasta la medianoche para anunciar la terrible noticia y 
trasladarlo a la Casa de la Cultura para velarlo. Lo ubican en una Hamaca blanca: la rellenan con telas simulando el muerto, la adornan con flores y la cuelgan en un ligero bambú para que puedan cargarla dos mujeres. A la media noche sale un hombre corriendo de la casa del difunto y rompe el precinto de seguridad cambiando la atmósfera del lugar, al mismo tiempo grita "Ya se murió" y todos responden "Hay que enterrarla" con el acompañamiento de los tambores, cachos, palas, picos y cualquier otro objeto como del hogar que emane sonido.

Segundo momento, El Velorio: Sale el difunto en la hamaca desde la Casa donde falleció y la gente se acerca a su encuentro. Detrás de ella y al ritmo de los tambores y con el canto de "Ya se murió", "Hay que enterrarla" llevan la Hamaca hasta la Casa de la Cultura donde esperan con las puertas abiertas entre luces de las velas. Allí la cuelgan y la gente se sienta alrededor, en orden las personas realizan sus peticiones y agradecen por los favores recibidos de años anteriores y colocan la vela encendida debajo de la Hamaca. Luego de estas peticiones algunos regresan a sus lugares $y$ otros amanecen entre bebidas y anécdotas sin baile. El difunto amanece resguardado en la Hamaca en la Casa de la Cultura.

Tercer momento, El entierro: A las doce del mediodía la gente se aglomera en la entrada de la Casa de la Cultura que permanece con las puertas cerradas, principalmente y en orden los hamaqueros quienes hacen un arco con las varas por donde pasará la Hamaca al salir de la Casa con sus cargadoras, promeseras, tanto las que custodian al muerto como las que esperan afuera. Los hombres hamaqueros se visten con un blue jean, alpargatas o deportivos, camisa floreada con un nudo al frente con el pecho semidesnudo, la vera para el combate y se colocan una cinta de color en la frente. Las mujeres llevan vestidos floreados con vuelo sutil por encima de las rodillas y de manga corta con alpargatas multicolores, también llevan la cinta de color en la frente o en el cabello y todos llevan la cara pintada de negro humo o pinturas de colores con unas rayas en los pómulos "por la libertad y resistencia". Justo a esa hora el Líder de la cofradía lee el decreto por los años que cumple la Hamaca y hacen el juramento de preservar por siempre la manifestación. Luego, vuelven a gritar "Ya se murió" y todos responden al unísono: "Hay que enterrarla" y abren las puertas y sale la Hamaca por el arco de veras (palos delgados y fuertes) y recorre todas las calles del pueblo con dos transiciones: (1) va al ritmo de "Ya se murió" con la respuesta de "Hay que enterrarla" entre tambores y cachos. Todas bailan al ritmo de una marcha y doble paso: las mujeres alrededor de la Hamaca formadas en una columna de cada lado y los hombres custodiando. Entre el recorrido cuando alguna otra mujer intenta cargar la Hamaca, un hombre golpea con su vera el bambú y hace caer la Hamaca al suelo, en ese momento las mujeres gritan desconsoladas, se tiran al piso a llorar y a gemir encima de la Hamaca. Cuentan algunos pueblerinos que el muerto era un picaflor y algún encuentro amoroso había tenido con las mujeres que lo rondaban y bailaban la Hamaca. (2) El llanto, gritos y gemidos de las mujeres genera celos en los hombres y ocasiona las peleas de veras de todos contra todos, hasta que ellas se levantan y hacen cambiar el ritmo musical por otro más alegre... los invitan a bailar para que dejen el combate, después levantan la Hamaca y los músicos retoman el primer ritmo con un tono lúgubre y de esta manera continuar el recorrido para enterrar al muerto hasta lograr su levantamiento. Durante el recorrido pintan a los que no están cubiertos con negro humo y pinturas de colores. Así 
recorren las calles del pueblo hasta llegar al malecón, hacen una parada y algunos se refrescan en la playa, después continúan hasta Rancho Grande hasta regresar a la Casa de la Cultura donde realizan el levantamiento.

Cuarto momento, El levantamiento: Al llegar todos los hamaqueros realizan un arco en un lateral de la Casa de la Cultura y las promeseras esperan con la Hamaca. Los hombres la toman y levantan hasta elevarla los más alto posible para poner fin y confiar en la "elevación" del alma del difunto con el Dios Altísimo Todopoderoso. $Y$ con agradecimientos concluye la celebración o rito hasta el siguiente año en los días de Carnaval.

Interrogante 2: ¿Podría narrar, brevemente, su experiencia si ha participado en un ritual semejante, además de describir sus pasos, momentos y asistentes?

Participé en esta grata y enriquecedora experiencia de la Hamaca por más de 5 años consecutivos, en el sector San Millán de Puerto Cabello del estado Carabobo, un barrio heredero de viviendas, tradiciones y costumbres afro, unido con algunas islas por el comercio y la tradición. Es un ritual semejante al de los alabaos por la dinámica, por ser expresión de nuestros pueblos, identidad cultural, costumbre y tradición que se transmite de generación en generación. Se corresponde con nuestros ciclos de fiesta tradicionales, es decir por el calendario festivo, se trata de una tradición ritual que también cumple unos momentos desde la muerte del difunto, el velorio, el entierro hasta el levantamiento. A esta celebración asisten adultos, jóvenes y niños de la localidad y también personas de todo el país y del exterior con intereses en investigar nuestras manifestaciones culturales de origen afro y de disfrutar de los carnavales, ya que, es parte de este ciclo o fiesta.
Interrogante 3: Desde su punto de vista como docente investigadora y artista creadora ¿cree que podrían incorporarse estas manifestaciones culturales en algunos de los niveles educativos y bajo qué tipo de acciones?

Si podrían incorporase en diversos niveles educativos como un estudio indispensable de la cultura, tradición y folclore de las localidades. Las acciones se pueden llevar en un proceso de sensibilización y adaptación de las manifestaciones culturales en el contexto educativo y hecho cultural de acuerdo con los contenidos que se desarrollan en cada nivel de estudio. Estas acciones como parte de la identidad cultural e idiosincrasia que se transmiten de generación en generación. Incentivando la experiencia desde el trabajo de campo y la investigación documental y generando proyectos para el desarrollo de actividades creativas se puede promover la enseñanza y aprendizaje de tales manifestaciones culturales.

\section{Entrevista a venezolana (2) Eva Nazareth Daza}

Interrogante 1: ¿Podría explicar si existe en su comunidad o región algún ritual semejante a los alabaos del Pacífico negro colombiano, así como sus pasos, momentos y asistentes?

Soy del pueblo de Curiepe, perteneciente a la Subregión Barlovento, del estado MirandaVenezuela. Existen rituales en torno a la muerte, pero no con las mismas estructuras del alabao, por ejemplo, cuando fallece algún cultor de la tradición, asistimos músicos y bailadores a cantar música Tradicional de Curiepe, toda la noche en el velorio, que acostumbra hacerse en la casa del difunto. En la puerta de la casa se coloca una cinta negra o morada como símbolo que esa familia está de luto y allí hay un difunto. Se reparte, aguardiente, café, cigarros y chocolate caliente. Al día siguiente, en el entierro, se 
canta y se toca, (tenemos la creencia de que los tambores tienen vida propia y un buen ejecutante es aquel que comprende la diferencia del tambor para la fiesta y el tambor fúnebre, este último que hace llorar a cualquier asistente). Luego se hacen las novenas, que consisten en rezos durante 9 noches, donde se reparte comida o merienda a los asistentes y finalmente "la última noche" o la última novena donde, obligatoriamente, se reparte chocolate caliente, galletas y queso salado.

Cuando muere un niño menor de 5 años se realiza un "mampulorio" o "velorio de angelito", se dispone un altar con el cuadro de la Virgen del Carmen, flores y velas; no se llora, porque el niño no va a pasar las penas de este mundo, se canta y se juegan juegos y rondas infantiles, y en un momento de la noche se canta "el mampulorio": "por las animas benditas que están en el purgatorio/ aquí está la vela- del mampulorio/ aquí está la olla- del mampulorio/ aquí está la tamboradel mampulorio.". En el entierro van niñas regando flores por las calles. Y se canta "yo vengo regando flores / por todo el camino real/ regálame tus amores/ para venirte a buscar".

Interrogante 2: ¿Podría narrar, brevemente, su experiencia si ha participado en un ritual semejante, además de describir sus pasos, momentos y asistentes?

Cuando tenía 14 años de edad asistí al último mampulorio que se realizó en Curiepe, me impresionó ver el altar y el niño difunto vestido de angelito sobre la mesa cuando llegó el momento de cantar el mampulorio, es difícil hacerlo sin que se quiebre la voz, aunque la gente mayor insiste en que no se debe llorar, para mí en aquel momento seguía siendo un niño muerto. Por lo demás, como música activa de mi pueblo, asisto en la medida de mis posibilidades a cantar $\mathrm{y}$ acompañar los velorios y entierros por compromiso y por amistad.

Interrogante 3: Desde su punto de vista como docente investigadora y artista creadora ¿cree que podrían incorporarse estas manifestaciones culturales en algunos de los niveles educativos $y$ bajo qué tipo de acciones?

Todos los elementos de la cultura popular tradicional son válidos como contenidos educativos, el aprendizaje es social, y llevar la tradición a la escuela es la garantía de la sistematización y masificación del aprendizaje. Por supuesto, a medida que se avanza académicamente ir profundizando en la información.

\section{Entrevista a venezolano (3) Yván Pineda Monasterios}

Interrogante 1: ¿Podría explicar si existe en su comunidad o región algún ritual semejante a los alabaos del Pacífico negro colombiano, así como sus pasos, momentos y asistentes?

Puedo decirle que sí. En Venezuela hay influencia africana, indígena y todo el legado de occidente; como somos un pueblo occidentalizado tenemos muchos rituales vinculados con la iglesia católica. Siendo un país pluricultural y multicultural el culto a los muertos define diferentes conductas dependiendo de la zona y la influencia de un grupo o de otro sea mayor. El culto a los muertos se divide en dos grandes momentos, el primero para confortar al difunto -aunque no están tan codificados como los alabaos de Colombia. Lógicamente, esos asentamientos del Pacíficos tienen grupos étnicos muy concentrados, mientras en el caso de Venezuela nosotros somos un pueblo muy mestizado y las tradiciones se han visto muy influenciadas por ese contacto. Entonces, hay rituales para la buena muerte, para bien morir 
hacen falta dos velas: la amarilla y roja de la Virgen de la Candelaria y la vela de la Primera Comunión. Estas velas se usan para ayudar en el tránsito hacia la luz, una anticipación y una manera de vincularse y aceptar el proceso de partida. Los indígenas Añú tienen un ritual que cuando un indígena se va a morir se mete en una hamaca y llama a una persona de su grande afecto para que le cante de tal forma que el moribundo se sube sobre la liviandad del canto y parte. Es una metáfora sublime del viaje. También entre los Añú la estera mortuoria es de mangle como mucho de lo que les rodea. Esta estera se coloca a secar al sol y luego de 9 días se supone que el muerto ya está del otro lado en donde le espera su familia y dos angelitos (a modo católico) que lo reciben con una fiesta. En este lado también hay una fiesta correspondiente. El difunto se queda complacido del sitio en donde llegó y los familiares del lado vivo ya lo pueden dejar ir en paz. El segundo momento se resumen en la frase: "por favor vete y no vuelvas más" se ayuda con agua, velas y rezos en los novenarios. Está por ejemplo "el cabo de ocho" de Barlovento.

Interrogante 2: ¿Podría narrar, brevemente, su experiencia si ha participado en un ritual semejante, además de describir sus pasos, momentos y asistentes?

Hay un ritual similar al de las tumbas en los Andes venezolanos en donde se construye el altar con los siete cielos con un motivo del gusto propio del difunto. El último día del novenario se cocinan hallacas, luego se hacen al año, a los 3 años y a los 7 años de la partida del difunto para cerrar el ciclo de los 7 cielos. En el lugar se canta y se pone la música favorita del difunto en un ambiente festivo y de armonía con comida por los asistentes por su regalo de haber permanecido sin dormir en la velada para el difunto.
Los indígenas tienen distintos ritos, en uno de ellos se cree que el difunto permanece en una especie de limbo porque aún no sabe qué está muerto, puede ocurrir entonces un segundo enterramiento dirigido por una mujer mayor de la familia, como el caso de los Guajiros o Wuayuu. También participé, con el grupo de los kariña: cuando se sabe que ya el difunto está listo para partir por un sueño de un familiar, se realiza una gran fiesta en la casa comunal y las mujeres danzan a manera de pájaros hasta que llega el ánima y comienzan las lamentaciones y las plañideras recorren toda la casa llorando y al final se hacen un corte de pelo con una totuma en la cabeza y al final se da la danza del Maremare para cerrar y el alma puede ir en paz.

También están "el mampulorio" y "la lombarda" cantos de lamentos en las regiones negras por la partida de un niño que parten sin haber manchado su alma con pecados y que esperarán en el cielo e intercederán ante Dios por sus padres y padrinos.

Interrogante 3: Desde su punto de vista como docente investigador y artista creador ¿cree que podrían incorporarse estas manifestaciones culturales en algunos de los niveles educativos y bajo qué tipo de acciones?

Es muy importante incorporar estas manifestaciones a los procesos educativos porque, por un lado, acercamos la educación a la vida de la cual ha estado algo desvinculada, mucho currículo y poco vitae; puede ayudar a comprender un proceso que es inevitable, un proceso que nos permite hacer duelo con los parientes y familiares; abrazar al niño o a la niña que pierde a su padre o su familiar, ser solidarios y empáticos con el otro; es un gran aprendizaje emocional trabajar en el aula y conversar acerca de esto. Se suma la parte musical, lo teatral, los contenidos sociales, los lazos de parentesco, 
como por ejemplo los padrinos de agua, de brazo, de luz. Entonces, es muy, muy importante incorporar esto a lo educativo y artístico porque el tratamiento tema de la muerte tanto en el arte como en la religión que son dos de los elementos que nos diferencian como seres humanos en tanto que somos la única especie que fabricamos símbolos de toda la creación. (Para escuchar la entrevista completa puede acceder haciendo click en este link: https://soundcloud.com/user380074600/representaciones-afroamericanas)

\section{Propuesta}

Luego de revisar la teoría y los contenidos de las entrevistas, se ofrece, una idea sobre la propuesta de inclusión del eje transversal Representaciones afroamericanas. Se sugiere incluir temas, experiencias, ejemplos de obras literarias, musicales y artísticas en el Componente Institucional Realidades y Tendencias Sociales y Educativas (Figura 1) en donde se encuentran las asignaturas Literatura Infantil y Juvenil, Literatura Colombiana, Literatura Latinoamericana, Lectura y Análisis de Textos Líricos y Dramáticos y Lógica y Argumentación.

En estos cursos, apropiadamente, podrían describirse, comentarse y analizarse los textos de los seis momentos del alabao (Tabla 2). Utilizando las muestras de los textos se puede comenzar por estudiar la literatura y la teoría del objeto literario; los límites del objeto centrado en la lengua; la función poética del lenguaje, la literariedad; la literatura y el signo lingüístico; la denotación y la connotación; describir el contenido temático, la ubicación en su temporalidad literaria, las características de la oratura y la oralitura, el contexto, la estructura lírica y estilística, los caracteres intertextuales. Igualmente se pueden realizar ejercicios de paráfrasis, análisis métrico, consideraciones sobre la historia de la literatura, conceptos de rima asonante y de rima consonante, conceptos de metáfora y símil, análisis de imágenes sensoriales, de otros recursos literarios como la humanización, la metáfora, la metonimia, la sinécdoque y el epíteto. (De la Torre Guerrero, 1988; Lázaro Carreter y Correa Calderón, 2006; Rodari, 2002; Selden, Widdowson, y Brooker, 2001; Sosa, Jesualdo, 1982; Subero, 2010; Tedesco, 2003; Viñas Piquer, 2002)

Asimismo, se pueden abordar expresiones cercanas a los alabaos como las retahílas, las coplas, las leyendas, los mitos y fantasmagorías, las narraciones históricas, las adivinanzas, los arrullaos o cantos de cuna en el curso de Literatura Infantil y Juvenil ; aprovechando, por una parte, el vínculo que este tipo de manifestaciones tienen con los procesos de oralidad que deben desarrollarse en la educación inicial y básica primaria y, de otro lado, que en la actualidad se cuenta con trabajos de antologías y recopilaciones que dan cuenta de representaciones propias de la tradición cultural del Pacífico colombiano (Revelo, 2010; Vanín, 2019), lo que hace más fácil su inclusión en los corpus de análisis. En cuanto al abordaje de la Literatura colombiana, el acercamiento al estudio de los alabaos, enriquecería de forma sustancial las lecturas que se hacen de obras que aborden temáticas referentes a las prácticas afro del país; a través de la comprensión de los procesos de sincretismo religioso presentes en los cantos fúnebres, las miradas alrededor de lo negro, en diálogo con las tradiciones africana, europea e indígena, ampliarían las miradas esencialistas que suelen tenerse sobre las obras afrocolombianas en las que lo humano se restringe a la hipersexualización de los sujetos, en especial de la mujer, al baile y a la gastronomía. 


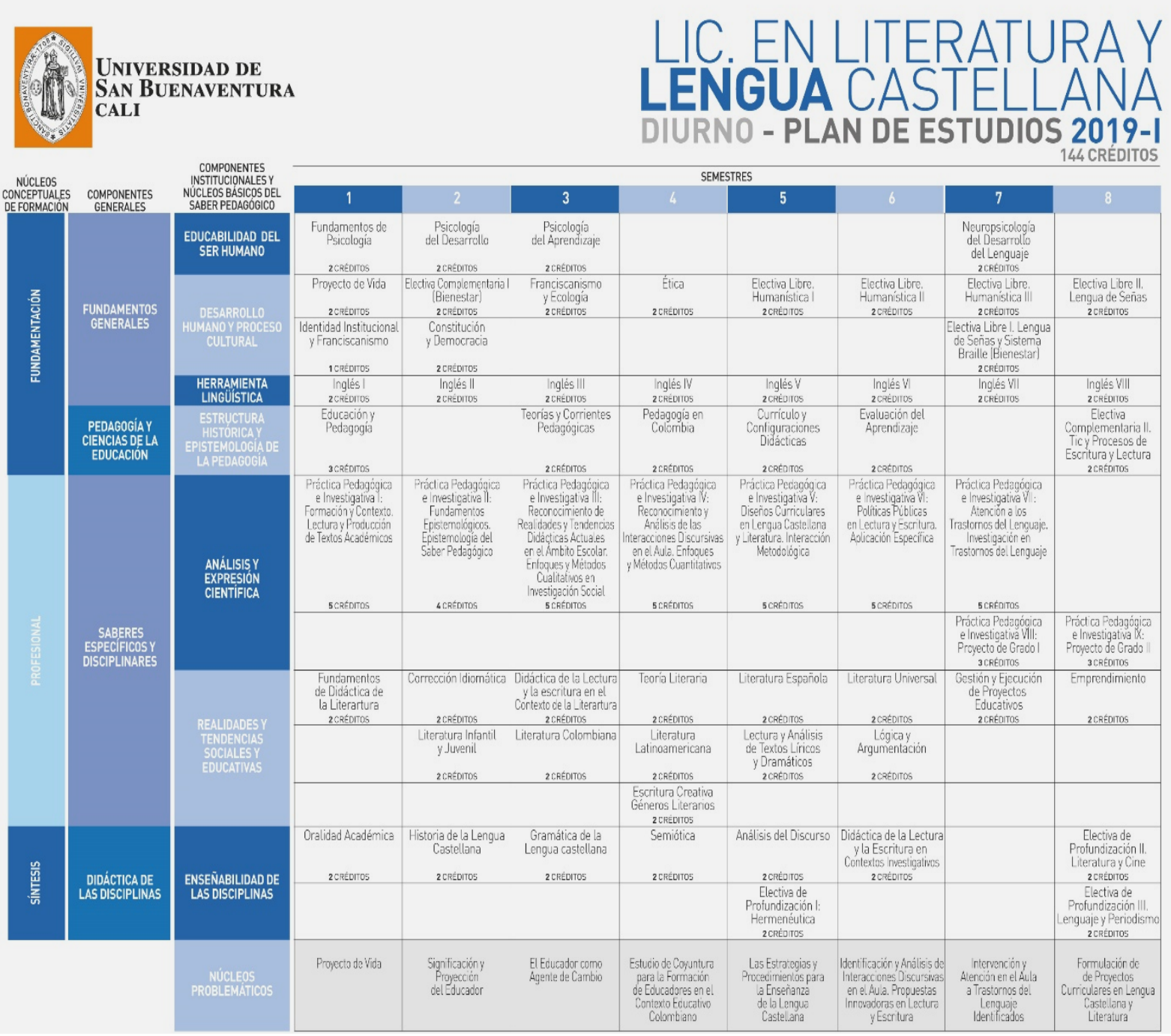

Figura 1. Plan de estudios LLLC. Universidad San Buenaventura de Cali.

Tabla 2. Ejemplos de estrofas de alabaos de sus seis momentos

\begin{tabular}{lll}
\hline $\begin{array}{c}\text { Rezo del primer momento: la } \\
\text { agonía: }\end{array}$ & $\begin{array}{c}\text { Rezo del segundo momento: la } \\
\text { muerte }\end{array}$ & $\begin{array}{c}\text { Rezo del tercer momento: } \\
\text { el velorio }\end{array}$ \\
\hline $\begin{array}{l}\text { Santo, Santo o Crisagio } \\
\text { Coro }\end{array}$ & $\begin{array}{l}\text { La muerte, cuando ella viene } \\
\text { (Versión Madolina Palacios) }\end{array}$ & $\begin{array}{l}\text { Alabao para el final del rosario } \\
\text { Dios te salve grandeza } \\
\text { Gloria al padre, Gloria al hijo, }\end{array}$ \\
$\begin{array}{ll}\text { El uno junto al otro } \\
\text { Gloria al Espíritu Santo. }\end{array}$ & $\begin{array}{l}\text { se aparta con gran dolor } \\
\text { Dios te salve brillante }\end{array}$ \\
Santo, Santo, Santo & se va callado la boca & luna en venida. \\
Señor Dios de los ejércitos & y a nadie le dice adiós & Cuando Dios en el mundo \\
llenos están los cielos & ni a nadie le da la mano. & hizo la arena. \\
y la tierra de la majestad & La muerte cuando ella viene & y se abrieron las aguas \\
inmensa de vuestra gloria. & no escoge buenos ni malos & cuando él la llena. \\
& tanto que éramos nosotros & Sintió Dios en el mundo \\
& padre, madre y hermanito. & hizo un semblante \\
& Y en la hora de mi viaje & y cesaron las aguas \\
en la menguante.
\end{tabular}




\begin{tabular}{ccc}
\hline $\begin{array}{c}\text { Rezo del primer momento: la } \\
\text { agonía: }\end{array}$ & $\begin{array}{c}\text { Rezo del segundo momento: la } \\
\text { muerte }\end{array}$ & $\begin{array}{c}\text { Rezo del tercer momento: } \\
\text { el velorio }\end{array}$ \\
\hline
\end{tabular}

\author{
$\mathrm{Y}$ aunque el gusano me coma \\ yo aquí debo volver \\ a recoger mis solare \\ que en este mundo dejé. \\ Que este mundo dejé \\ en el otro lo encontré \\ que lo tenía la virgen \\ pa' el glorioso San José.
}

Dios te salve brillante luna en venida. Dios te salve María llena de gracia y el Señor de los cielos entró a esta casa.

\section{Rezo del cuarto momento: el entierro}

\section{Entierren al que se muere \\ Coro}

Entierren al que se muere

lo manda la ley cristiana

que lo metan a la iglesia

y doblen todas las

campanas.

Cuando un pecador se

muere

qué consejo le darán

acá no se necesita

alma sin saber rezar.

Coro

Cuando un pecador se

muere

qué consejo le darán

a cantarle a la virgen

y a la Trinidad.

Coro

Señora me confesé

porque no podía sabé

con qué me podía salvá.

\section{Rezo del quinto momento: la} novena

Santo Días y Santo Fuerte y adoremos un Dios Divino Jesucristo está enclavado y en un amargo camino Con la hostia consagrada convertida en pan y vino (Se dice tres veces)

\section{Rezo del sexto momento: la} última noche

En el nombre de María

hijo de Jesús en Belén

un San Antonio

y un Cristo también

Con las ánimas benditas

descansen en paz

Y en el nombre de María

Santísima Trinidad

levanten la tumba

que esta alma se va.

Levanten la tumba

del cuerpo presente

se despide esta alma

en vida y en muerte.

Nueve noches son

las de mi novena

levanten la tumba

que esta alma es ajena.

El que esté llorando

déjenlo llorar

que estos son los golpes

que mi Dios nos da.

"Levanten la tumba

del cuerpo presente

se va un pecador

en vida y en muerte".

"Aquí se despide este pecador

ha dejado este mundo

por servirle a Dios".

"Adiós mi primo hermano, primo hermano adiós, te vas y nos dejas solitos con Dios".

Fuente: recopilación propia 


\section{CONCLUSIONES}

A pesar de los esfuerzos que desde el gobierno central se han movilizado para el reconocimiento y estudio de las prácticas culturales de la población afrodescendiente en Colombia, visibles en propuestas como la Cátedra de Estudios Afrocolombianos (1998) y el documento Colombia Afrodescendiente. Lineamientos Curriculares de la Cátedra de Estudios Afrocolombianos (2010) este ejercicio sigue evidenciándose en prácticas aisladas de docentes e Instituciones Educativas. Por eso, como bien se consiguió recoger en las voces de los especialistas entrevistados, movilizar desde los procesos de formación profesoral el reconocimiento y estudio riguroso de este tipo de manifestaciones, permitirá, reflexionar sobre los procesos identitarios implicados y motivar, para que, una vez en su ejercicio profesional, los integren a sus planes curriculares. Todo esto toma una mayor relevancia si se tiene en cuenta que alrededor del $50 \%$ de los habitantes de la ciudad de Cali son afrodescendientes y que esta cifra la ubica como una de las ciudades latinoamericanas con el mayor número de población negra.

La conjunción que al interior de estas composiciones cantadas se configura a través de la presencia de la poesía española popular, la influencia indígena y los saberes ancestrales africanos que llegaron a América gracias a la enorme capacidad mnemotécnica de los hombres esclavizados abre múltiples posibilidades de estudio desde la transversalidad disciplinar, estudios intertextuales y la problematización de los cánones que históricamente se han impuesto en la escuela y que siguen perpetuando prácticas de invisibilización de poblaciones que han sido marginadas. Todos estos factores aportarían de forma sustancial a la formación de los especialistas en el estudio de la literatura y la lengua castellana en los contextos colombiano y latinoamericano.

\section{REFERENCIAS}

Amézquita, A. y Hernández, C. (2012). Alabaos: narraciones de una comunidad. Relatos de vida y muerte. (Tesis de Maestría). Universidad del Valle, Cali, Colombia

Amézquita, A. y Hernández, C. (2013). Alabaos, narraciones de vida y muerte de una comunidad. Revista Phoenix, 14. $42-51$

Ayala Santos, A. G. (2010). Amuletos y Santos en el Atrato. Medellín, Colombia: Editorial Mundo Libro

Córdoba Cuesta, D. y Rovira de, C. (2003). El Alabao. Cantos fúnebres de la Tradición Oral del Pacífico Colombiano. Bogotá, Colombia: Corporación Identidad Cultural

Córdoba, E. (2014). El papel de la décima espinela en la cultura latinoamericana. La Clé des Langues [en ligne], Lyon. Recuperado de http://cle.enslyon.fr/espagnol/litterature/litteraturelatino-americaine/poesie/el-papel-de-ladecima-espinela-en-la-culturalatinoamericana

Corporación Centro de Pastoral Afrocolombiana. CEPAC. (2010). Tradiciones Religiosas Afrocolombianas. Celebrando la Fe desde la Cultura. Popayán, Colombia: CEPAC

De la Torre Guerrero, G. (1988). Nuestro Alabao: notas sueltas sobre un tema clave. Revista Por la Vida, 2

Friedemann, N. (1992). Huellas de africanía en Colombia. Nuevos escenarios de investigación. Thesaurus. Boletín del Instituto Caro y Cuervo. Tomo $X L V I I, 3$, 543-560. Recuperado de https://cvc.cervantes.es/lengua/thesauru s/pdf/47/TH_47_003_071_0.pdf

Friedemann, N. (1997). De la tradición oral a la etnoliteratura. América Negra. 13, 119

González, J. (1995). Actores sociales y dinámicas culturales de la religiosidad popular negra en algunas localidades del Pacífico colombiano: análisis de testimonios. Cali, Colombia: Universidad del Valle, Escuela de Comunicación Social, Facultad de Artes Integradas 
Lázaro Carreter, F. y Correa Calderón, E. (2006). Cómo se comenta un texto literario. Madrid: Cátedra

Losonczy, A. M. (2006). La trama interétnica. Ritual, sociedad y figuras de intercambio entre los grupos negros y Emberá del Chocó. Bogotá, Colombia: Instituto Colombiano de Antropología e Historia. Instituto Francés de Estudios Andinos

Moreno Blanco, J. (2008). Corpus y cánones de la oralidad cultural en la educación en Colombia. Una mirada introductoria. Folios. Segunda época. Revista de la Facultad de Humanidades Universidad Pedagógica Nacional, 27, 90-96. Recuperado de https://www.redalyc.org/pdf/3459/34594 1357008.pdf

Motta González, N. (1997). Hablas de Selva y Agua. La oralidad afropacífica desde una perspectiva de género. Cali, Colombia: Universidad del Valle, Centro de género, mujer y sociedad. Instituto de Estudios del Pacífico

Prado, N. (1996). El origen de los versos para enamorar: oralidad del Pacífico del sur de Colombia. En América Negra. Expedición humana a la zaga de la América oculta, $12 . \quad 195-214$. Recuperado de https://www.javeriana.edu.co/documents /5782625/5900951/AmericaNegra12.pdf/ b2fcbfcf-764c-40e6-999c-a9fac9313c4c

Puertas Arias, E. (2000). Del Pacífico colombiano. La tunda. Mito y realidad. Sus funciones sociales. Cali, Colombia: Línea Cultura

Revelo, B. (2010). Cuentos para dormir a Isabella: tradición oral afropacífica colombiana. Bogotá, Colombia:
Ministerio de Cultura de Colombia, Biblioteca de literatura afrocolombiana

Rodari, G. (2002). Gramática de la fantasía. Introducción al arte de contar historias. Barcelona: Planeta

Ruay, R., González, P. y Plaza, E. (2016). ¿Cómo abordar la renovación curricular en la educación superior? Revista de Educación Alteridad, 11(2), 157-170. Recuperado de http://dx.doi.org/10.17163/alt.v11n2.201 6.02

Selden, R., Widdowson, P. y Brooker, P. (2001). La teoría literaria contemporánea. Barcelona, España: Ariel

Sosa, Jesualdo. (1982). La literatura infantil. Buenos Aires: Losada

Subero, E. (2010). La décima popular en Venezuela. Caracas, Venezuela: Fundación Bigott

Tedesco, Í. (2003). Urdimbre estética, social e ideológica del indigenismo en América Latina. Caracas, Venezuela: Ediciones del Vicerrectorado de Investigación y Postgrado

Tobón, A. (2010). Romances religiosos: de la España medieval a los rituales negros en el Atrato. En Sevilla, M. Santamaría, C. y Ochoa, J. (editores) Músicas y prácticas sonoras en el Pacífico afrocolombiano. Bogotá, Colombia: Editorial Pontificia Universidad Javeriana

Vanín, A. (2019). Mito, tradición oral, historia y literatura del Pacífico colombiano, Antología. Cali, Colombia: Fondo de Publicaciones del Valle del Cauca

Viñas Piquer, D. (2002). Historia de la crítica literaria. Madrid: Ariel 